\title{
Edwardsiella ictaluri invasion of IEC-6, Henle 407, fathead minnow and channel catfish enteric epithelial cells
}

\author{
Ramona T. Skirpstunas*, Thomas J. Baldwin \\ Department of Veterinary Microbiology and Pathology, Washington State University, Pullman, Washington 99164, USA
}

\begin{abstract}
Invasion of Edwardsiella ictaluri into cultured mammalian, fish and enzymatically harvested catfish enteric epithelial cells is described. Gentamicin survival assays were used to demonstrate the ability of this catfish pathogen to invade IEC-6 (origin: rat small intestinal epithelium), Henle 407 (origin: human embryonic intestinal epithelium), fathead minnow (FHM, minnow epithelial cells) and trypsin/pepsin-harvested channel catfish enteric epithelial cells. Invasion of all cell types occurred within $2 \mathrm{~h}$ of contact at $26^{\circ} \mathrm{C}$, in contrast to Escherichia coli DH5 $\alpha$, which did not invade cells tested. Eight Edwardsiella ictaluri isolates from diseased catfish and the ATCC (American Type Culture Collection) strain were evaluated for invasion efficiency using FHM cells. All isolates were invasive, but at differing efficiencies. Invasion blocking assays using chemical blocking agents were performed on a single isolate (LA 89-9) using IEC-6 epithelial cells. Preincubation of IEC-6 cells with cytochalasin D (microfilament depolymerizer) and monodansylcadaverine (blocks receptor-mediated endocytosis) significantly reduced invasion by E. ictaluri, whereas exposure to colchicine (microtubule depolymerizer) had no effect on bacterial internalization. Results indicate that actin polymerization and receptor-mediated endocytosis are involved in uptake of E. ictaluri by IEC-6 epithelial cells. Invasion trials using freshly harvested cells from the intestine of the natural host, Ictalurus punctatus, show that invasion occurs, but at a low efficiency. This is possibly due to loss of outer membrane receptors during enzymatic cell harvest. This study provides the first documentation of the invasion of cultured mammalian and fish cells by E. ictaluri, and identifies possible mechanisms used for intracellular access. Additionally, the study describes several functional in vitro invasion models using commercially available cell lines as well as cells from the natural host (channel catfish, I. punctatus).
\end{abstract}

KEY WORDS: Edwardsiella ictaluri $\cdot$ Enteric septicemia of catfish $\cdot$ ESC $\cdot$ Catfish diseases

\section{INTRODUCTION}

Edwardsiella ictaluri is a Gram-negative, rodshaped bacterium in the family Enterobacteriaceae (Hawke 1979, Hawke et al. 1981). It is the causative agent of enteric septicemia of catfish ( $\mathrm{ESC}_{i}$ Hawke 1979, Hawke et al. 1981), the most economically important bacterial disease in farmed channel catfish, Ictalurus punctatus. Enteric septicemia of catfish

*Present address: Vet Diagnostic Laboratory, ADVS Department, Utah State University, 5700 Old Main Hill, Logan, Utah 84322, USA. E-mail: rskirps@cc.usu.edu accounts for approximately $60 \%$ of all mortality in farmed channel catfish, which results in approximately 50 million dollars in annual losses (Mitchell 1997). Diseased fish either become acutely septic or develop chronic meningoencephalitis, both of which contribute to significant losses. The lack of effective vaccines and recent development of resistance to the few antibiotics approved for use in food fish (Shotts et al. 1985, Plumb \& Vinitnantharat 1990, Cooper 1996) have greatly hindered efforts to control ESC.

Entry of Edwardsiella ictaluri into channel catfish occurs through epithelial-lined tissues, including gastrointestinal tract and olfactory mucosa (Blazer et al. 
1985, Shotts et al. 1985, Saeed \& Plumb 1986, Newton et al. 1988, Baldwin \& Newton 1993), with no histologic evidence of epithelial cell damage. This suggests that E. ictaluri traverses the epithelial lining by exploiting normal cellular transport systems. Once past the epithelial barrier, proprial macrophages are thought to take up the bacterium, which is then transported systemically via the bloodstream (Miyazaki \& Plumb 1985). Sepsis and death often ensue.

Detailed molecular studies of initial interactions of Edwardsiella ictaluri with susceptible cells are lacking, in part due to the absence of a functional in vitro model. Live fish models (enteric inoculation) have inherent limitations, such as extensive surface area and mixed cell populations, whereas cultured cell models allow for a detailed look at initial interactions between bacterial organisms and specific cell types. Consequently, models utilizing cultured cells are critical for identification of specific molecules and/or mechanisms used in initial bacteria/host cell interactions. In cases where a susceptible cultured cell line is not available, epithelial cells harvested from host tissues known to support invasion can substitute as assay systems.

Efforts toward identification of possible mechanisms of cell invasion used by Edwardsiella ictaluri have, until now, relied solely on live fish models, since cultured cell systems susceptible to E. ictaluri invasion have not been previously identified. This paper documents invasion of E. ictaluri into IEC-6 (rat small intestinal epithelium), Henle 407 (human embryonic intestinal epithelium), and fathead minnow (minnow epithelial cells) cell lines using gentamicin survival assays. In addition, invasion of trypsin/pepsinharvested catfish enteric epithelial cells is described.

Entry of many invasive enteric bacteria into eukaryotic cells involves exploitation of existing cellular pathways (Finlay \& Falkow 1988, 1989, Finlay et al. 1989). Exploiting receptor-mediated endocytosis or triggering pinocytosis using specific cell-surface receptors are methods commonly used by other pathogenic members of the Enterobacteriaceae to enter host cells (Miller et al. 1988, Finlay \& Falkow 1989, Finlay et al. 1989). Cultured cell-invasion models and invasionblocking assays employing chemical inhibitors of specific host cytoskeletal or surface elements are used to help define these initial events (Finlay \& Falkow 1988, 1989, Oeschlager et al. 1994). Cytochalasin D is an actin depolymerizer that inhibits host-cell microfilament functions necessary for active uptake of extracellular products. Colchicine depolymerizes microtubules and inhibits transport and movement of intracellular vesicles within host cells. Monodansylcadaverine (MDC) is a transglutaminase inhibitor that deactivates receptor-mediated endocytosis (Pastan \& Willingham
1981). This study reports on the mechanisms involved in the uptake of Edwardsiella ictaluri determined using these 3 inhibitors of eukaryotic cytoskeletal function. The IEC-6 cells were chosen as the pilot cell line for blocking assays because of the adaptability of these cells to specific assay conditions and because they have been used in similar (mammalian) invasionblocking assays.

Finally, pepsin/trypsin harvested enteric epithelial cells were employed as an invasion system using gentamicin survival assays (Ellinghorst 1994). Results show consistent invasion of cells by Edwardsiella ictaluri, although the invasion efficiencies are lower than those described in cultured cell lines tested. Nevertheless, these findings provide another alternative in vitro cell system for the study of E. ictaluri invasion and provide a functional technique for procurement of susceptible cells from the natural host, providing for future studies involving host-cell receptors.

\section{MATERIALS AND METHODS}

Cell lines and culture conditions. IEC- 6 cells (rat small intestinal epithelial cell line CRL-1592, ATCC) were grown and maintained in minimal essential medium (MEM; Gibco/BRL Life Technologies) containing $5 \%$ heat-inactivated fetal bovine serum (HyClone Laboratories), $2 \mathrm{mM}$ glutamine (Gibco/BRL) and $0.1 \mathrm{U} \mathrm{ml}^{-1}$ bovine insulin (Sigma Chemical). The Henle 407 cell line (human embryonic intestinal epithelial cells, CCL-6, ATCC; kindly provided by P. Mixter, Washington State University, Pullman) were grown and maintained in Dulbecco's modified Eagle's media (DMEM; Gibco/BRL) containing 10\% heatinactivated calf serum (HyClone) and Hank's basic salt solution (Gibco/BRL). Fathead minnow (FHM) cells were grown in modified Eagle's media (MEM; Gibco/BRL) containing 10\% heat-inactivated fetal bovine serum. Stock cell cultures were maintained in $75 \mathrm{ml}$ culture flasks (Becton Dickinson Labware) in antibiotic-free media at $37^{\circ} \mathrm{C}$ in $5 \% \mathrm{CO}_{2}$ (IEC-6 and Henle 407 ) or $30^{\circ} \mathrm{C}$ (FHM) in normal atmosphere, and split weekly. Monolayers for invasion assays were prepared by seeding approximately $2.5 \times 10^{3}$ cells in $1 \mathrm{ml}$ growth medium in each well of a 24-well tissueculture plate (Falcon Primaria, Fischer Scientific, Pittsburgh, Pennsylvania). Confluent monolayers were obtained after $72 \mathrm{~h}$. Immediately before performing invasion assays, each medium was removed from each well and replaced with $1.0 \mathrm{ml}$ of fresh medium, and cells were subsequently allowed to acclimate to $26^{\circ} \mathrm{C}$ for several hours in normal atmosphere. 
Intestinal cell suspension preparation. Intestinal segments were harvested from juvenile (minimum 8 $\mathrm{cm}$ long) channel catfish after terminal exposure to tricaine methanesulfonate (MS-222, Argent Chemical Laboratories) dissolved at $5 \%$ concentration in dechlorinated tap water. Intestines were opened length-wise and gently washed in calcium and magnesium-free phosphate buffered saline (CMF, $\mathrm{pH} 7.2$ ) for $2 \mathrm{~min}$ to remove the surface mucus. After 3 washes in fresh $\mathrm{CMF}$, intestinal segments were incubated in ice-cold CMF containing $0.25 \%$ trypsin plus $1 \%$ pepsin on a rocker for $60 \mathrm{~min}$. After incubation, tissue fragments were triturated using a large-bore pipette tip to release connective tissue and muscle from mucosal cells. Large tissue fragments were allowed to settle and were removed manually. Suspended cells were centrifuged at $500 \times g$ for 4 min and washed with L-15 medium (Gibco/BRL) 3 times before resuspension in 1 $\mathrm{ml}$ volumes of L-15 medium. Samples from each tube were applied to a hemacytometer and the approximate cell numbers $\mathrm{ml}^{-1}$ were determined.

Cell viability and counts. The viability of IEC-6, Henle 407 and FHM cells was determined by $0.4 \%$ trypan blue exclusion before and after bacterial exposure, just prior to lysis and after incubation with chemical inhibitors (IEC-6 cells). In all cases, cells showed $<1 \%$ dye incorporation, indicating maintenance of membrane integrity throughout each assay.

The number of cells per well was estimated before each assay by counting cells in 2 wells of the culture plate using a calibrated grid and an inverted light microscope. Cells were counted to determine the bacterial:epithelial cell ratio (multiplicity of infection, MOI) used during each invasion assay. Cell cultures were tested for the presence of contaminating bacteria by plating lysed cells from negative control wells before each experiment. Cell counts of intestinal cell suspensions prior to addition of bacteria were performed using a hemacytometer. Cell viability was determined using $0.4 \%$ trypan blue exclusion. No cell staining was detected before bacterial incubation and $<1 \%$ of cells stained positively after gentamicin incubation.

Bacteria. Stock cultures of 9 separate isolates of Edwardsiella ictaluri (LA-89-9, 93-146, 01-101: Joseph Newton, Auburn University, Auburn, Alabama; 324, 492, 513 and 551: Washington Animal Disease Diagnostic Laboratory, Pullman, Washington; 95-135: John Hawke, Louisiana State University, Baton Rouge, Louisiana; and ATCC Strain 669), and single isolates of Yersinia enterocolitica (ATCC 19873897-04) and Escherichia coli DH5 a (ATCC 53868) were obtained, amplified in brain heart infusion broth (BHIB, Becton Dickinson) and stored at $-80^{\circ} \mathrm{C}$ in BHIB with $20 \%$ glycerol. To ensure virulence of E. ictaluri, channel cat- fish fingerlings were orally inoculated with approximately $1.0 \times 10^{9}$ colony-forming units (CFU) per fish and trunk kidneys were cultured on selective media (Shotts \& Waltman 1990) $24 \mathrm{~h}$ post-infection. Resulting bacteria were used to orally re-infect other fingerlings, and only bacteria recovered from kidney cultures were used in cell-invasion assays.

Single colonies were confirmed as Edwardsiella ictaluri by microagglutination using polyclonal rabbit anti whole-cell E. ictaluri antisera (Micrologix International). Yersinia enterocolitica was used as a positive control in invasion and invasion-blocking assays, and Escherichia coli DH5 $\alpha$ (non-invasive) was used as a negative control. Stock cultures of $Y$. enterocolitica were grown overnight in BHIB broth at $26^{\circ} \mathrm{C}$ while $E$. coli DH5 $\alpha$ was grown for $12 \mathrm{~h}$ at $37^{\circ} \mathrm{C}$. Bacteria were harvested by centrifugation at $2500 \times g$ for $10 \mathrm{~min}$ at $4^{\circ} \mathrm{C}$ and washed twice using $10 \mathrm{ml}$ of sterile CMF before resuspension in CMF to the desired colony-forming units (CFU) ml ${ }^{-1}$ concentration. Colony-forming unit counts were verified by dilution plating of each bacterial suspension onto BHI agar (Becton Dickinson).

Invasion assays. Invasion assays were performed using standard in vitro methodology: bacteria were incubated with epithelial cells followed by selective killing of extracellular bacteria by gentamicin and subsequent release of internalized bacteria by lysis of epithelial cells with a detergent solution (Rubens et al. 1992, Ellinghorst 1994). Invasion assays of cultured cells were performed in 24 well plates (Falcon Primaria) by incubating Edwardsiella ictaluri, Yersinia enterocolitica or Escherichia coli DH5 $\alpha$ organisms with confluent IEC-6, Henle 407 or FHM cells for 2.5 to $3 \mathrm{~h}$ (invasion period) at $26^{\circ} \mathrm{C}$ in normal atmosphere. The medium was removed and cells were washed twice with CMF. Media containing $100 \mu \mathrm{g} \mathrm{ml}^{-1}$ gentamicin (Gibco/BRL) was applied and cells incubated for $4 \mathrm{~h}$ at $26^{\circ} \mathrm{C}$ (extracellular killing period). Cells were washed 4 times with CMF and $100 \mu$ of the final wash were plated onto BHI agar or E. ictaluri-selective media to ensure effective killing/removal of extracellular bacteria. Trypan blue was added to wells and viable cell counts were performed prior to cell lysis. The fluid overlying the cells was removed and the cells were lysed with $200 \mu \mathrm{l}$ of $1 \%$ Triton $\times 100$ (Amersham, Arlington Heights, Illinois) in CMF. After 10 min, samples of the cell lysate were triturated using a transfer pipette, serially diluted, plated on BHI or E. ictaluriselective media (Shotts \& Waltman 1990), and incubated at $26^{\circ} \mathrm{C}$ for intracellular $\mathrm{CFU}$ determination. Cells that were incubated with bacteria but that did not receive gentamicin ensured bacterial viability throughout the experiment. Cells that were taken through all assay steps except that of having bacteria added ensured that cultured cells were free of intracel- 
lular bacteria, and that cells were not damaged by washing or antibiotic treatments.

Cell suspension invasion assays were performed similarly, with the exception that $1.5 \mathrm{ml}$ microcentrifuge tubes were used, and washes and media changes were followed by pelleting cells at $600 \times g$ for 4 min after which the cells were resuspended. Intestinal cell suspensions were washed and maintained in L15 medium throughout the assay. Gentamicin at $100 \mu \mathrm{g} \mathrm{ml}^{-1}$ was added to L-15 for the extracellular killing period. All treatments were identical to those described above, except that only Edwardsiella ictaluri and Escherichia coli invasion were evaluated.

Effects of inhibitors and DMSO on IEC-6 cells and Edwardsiella ictaluri. Cytopathic effects of cytochalasin D, colchicine, MDC and dimethylsulfoxide (DMSO, solubilization agent for cytochalasin D and MDC) were determined in preliminary experiments to optimize assay conditions and standardize inhibitor concentrations for use in invasion-blocking experiments. Effects on bacteria were measured using CFU/ml counts. Effects on epithelial cells were measured using trypan blue exclusion. Exposure times were equivalent to those of a standard invasion assay (2.5 to $3 \mathrm{~h}$ ). Highest concentrations having no cytotoxic effects were used (cytochalasin D at $2 \mu \mathrm{M}$, colchicine at $10 \mu \mathrm{M}$ and MDC at $125 \mu \mathrm{M})$. Since DMSO is a transmembrane carrier at high concentrations, the effect of $1 \%$ DMSO on bacterial internalization was also examined. Pretreatment of cells with $1 \%$ DMSO had no effect on numbers of internalized bacteria compared with non-treated controls (data not shown). These results ensure that decreases in numbers of internalized bacteria in the blocking experiments were due to

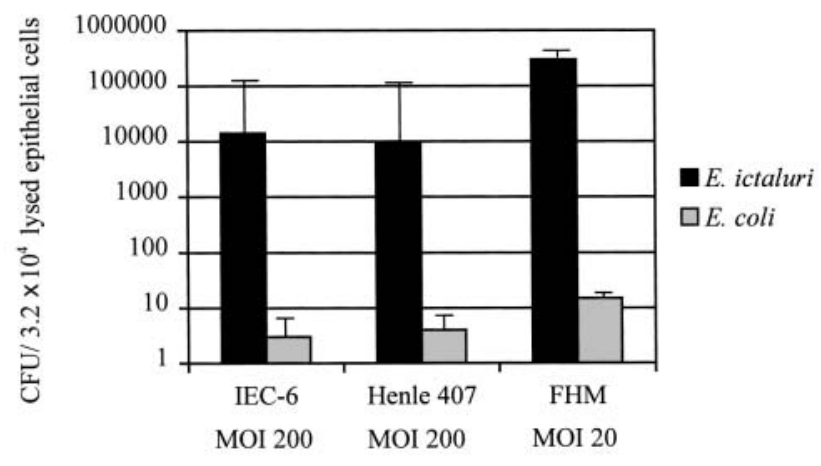

Fig. 1. Invasion susceptibility of IEC-6, Henle 407 and FHM cell lines to Edwardsiella ictaluri (Isolate LA89-9). Bacterial suspensions were incubated with cultured cell monolayers for $2 \mathrm{~h}$ at $26^{\circ} \mathrm{C}$, after which extracellular bacteria were killed by incubation of cells in media containing gentamicin. The mean and standard error of colony-forming units (CFU) per well from 3 separate trials using 3 well replicates is shown. Error bars compare the standard error of the mean of each of the 3 trials modification of cytoskeletal elements or endocytic processes as opposed to cytotoxic or bacteriocidal effects of inhibitors.

Invasion-blocking assay (IEC-6 cells). Inhibitors were dissolved in distilled water (colchicine) or $1 \%$ DMSO solution (cytochalasin D and MDC), divided into aliquots, and stored at $-20^{\circ} \mathrm{C}$. Invasion inhibitors were added to the medium overlying IEC- 6 cells for $1 \mathrm{~h}$ at $26^{\circ} \mathrm{C}$ immediately prior to performing invasion assays. Bacteria were incubated with the pretreated monolayers for $2 \mathrm{~h}$ at $26^{\circ} \mathrm{C}$ and each well was rinsed 4 times with clean DMEM containing 5\% FBS. Gentamicin exposure, cell lysis and bacterial enumeration were performed as described previously.

Statistics. All invasion and blocking assays were performed in triplicate and experiments were repeated independently 3 times. All data were expressed as mean and standard error of the mean (SEM) or standard deviation (SD). Data in blocking assays were compared with non-treated controls using Student's $t$ test with a level of significance at $p \leq 0.05$.

\section{RESULTS}

IEC-6, Henle 407, FHM cells, and harvested catfish enterocytes were susceptible to invasion by Edwardsiella ictaluri. Maximum internalization of E. ictaluri (LA 89-9) in IEC-6 cells occurred at an MOI of approximately 200 bacteria per epithelial cell. In FHM cells, maximum internalization occurred at an MOI of 40 bacteria per epithelial cell. The efficiency of $E$. ictaluri internalization by the 3 cell lines expressed as total CFU per monolayer is shown in Fig. 1. Although all 3 cell lines were susceptible to invasion, direct comparisons of efficiencies cannot be made due to variability in MOI. Nevertheless, invasion efficiencies in IEC-6 and Henle 407 cells were clearly lower than those for FHM cells. Experiments using harvested intestinal epithelial cells showed internalized numbers of bacteria to be low, and successful invasion required an MOI of 100 to 200 . Colony-forming units per $1 \mathrm{ml}$ of total cell lysate are shown in Fig. 2.

Yersinia enterocolitica and Escherichia coli DH5 $\alpha$ were run concurrently in invasion and blocking trials, as positive and negative controls, respectively. Y. enterocolitica cells were internalized consistently in high numbers (data not shown) and were used to ensure epithelial cell susceptibility to a known invasive organism. E. coli cells were used to control against nonspecific internalization in all cell lines tested.

Since IEC-6 cells were easily adaptable to assay conditions (incubation periods, chemical treatments and temperature) they were chosen as the pilot line for invasion-blocking assays. IEC-6 cells treated with 
Table 1. Edwardsiella ictaluri. Percent relative invasiveness. Values are percent of bacteria internalized into IEC-6 cells pretreated with chemical blockers compared to total colonyforming units of internalized E. ictaluri (LA 89-9) in nontreated IEC-6 cells (considered as 100\% invasion). Values are the mean and standard error of mean of 3 experiments performed in triplicate. ${ }^{*}$ Significant difference $(p \leq 0.05)$ compared to non-treated controls

\begin{tabular}{|cccc|}
\hline Non-treated & Cytochalasin & Colchicine & MDC \\
\hline 100 & $0.04 \pm 0.02^{*}$ & $89.7 \pm 0.19$ & $0.03 \pm 0.03^{*}$ \\
\hline
\end{tabular}

cytochalasin D $(2 \mu \mathrm{M})$ showed slight morphologic changes including cell rounding and mild cytoplasmic contraction compared to untreated cells. Colchicine $(10 \mu \mathrm{M})$ and MDC $(125 \mu \mathrm{M})$ treatments did not alter cell morphology. Trypan blue exclusion showed maintenance of membrane integrity of more than $99 \%$ of cells throughout all experiments and treatments.

Uptake of Yersinia enterocolitica by IEC-6 cells was significantly decreased by preincubation of cells with cytochalasin D and MDC, while no significant effect on uptake was seen with colchicine pretreatment (data not shown). Likewise, pretreatment of IEC-6 cells with cytochalasin D reduced invasion of Edwardsiella ictaluri by $99.96 \%$ compared to similarly exposed untreated cells (Table 1). Pretreatment of cells with colchicine had no significant effect on E. ictaluri internalization (Table 1). Pretreatment of IEC-6 cells with monodansylcadaverine reduced invasion of E. ictaluri by $99.97 \%$ (Table 1$)$.

Variability in invasion efficiency between various cell lines and the harvested cell preparation using Isolate LA 89-9, initiated an effort to determine if invasiveness was strain-related. Gentamicin survival assays were concurrently run using 8 different isolates of Edwardsiella ictaluri on FHM cells. Results showed all isolates of $E$. ictaluri tested were invasive, with some isolates having greater efficiency using this assay system. Colony forming units per monolayer of FHM cell lysate are shown in Fig. 3.

\section{DISCUSSION}

Using gentamicin survival assays, this study documents that IEC-6, Henle 407 and FHM cell lines, as well as freshly harvested intestinal cell suspensions from channel catfish, are susceptible to invasion by Edwardsiella ictaluri. This study presents the first in vitro data supporting intracellular invasion by E. ictaluri, and identifies 4 viable epithelial cell invasion models for future studies. In vitro models are necessary to study the molecular pathogenesis of ESC, which is largely un-

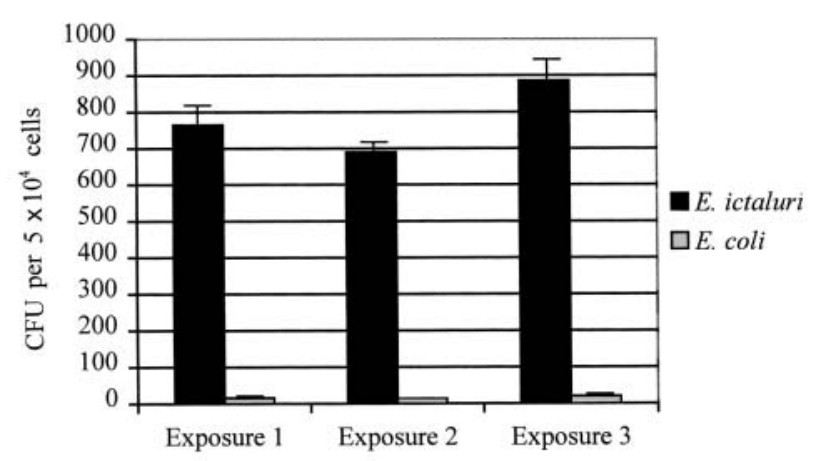

Fig. 2. Colony-forming units (CFU) per $1 \mathrm{ml}$ of total cell lysate (trypsin/pepsin-harvested intestinal epithelial cells of channel catfish) of approximately $5 \times 10^{4}$ cells after incubation with Edwardsiella ictaluri (multiplicity of infection, MOI 100 to 200) for $2 \mathrm{~h}$ at $26^{\circ} \mathrm{C}$, followed by a $4 \mathrm{~h}$ incubation in media containing $100 \mu \mathrm{g} \mathrm{ml}^{-1}$ gentamicin. Columns represent means of 3 independent experiments performed in duplicate

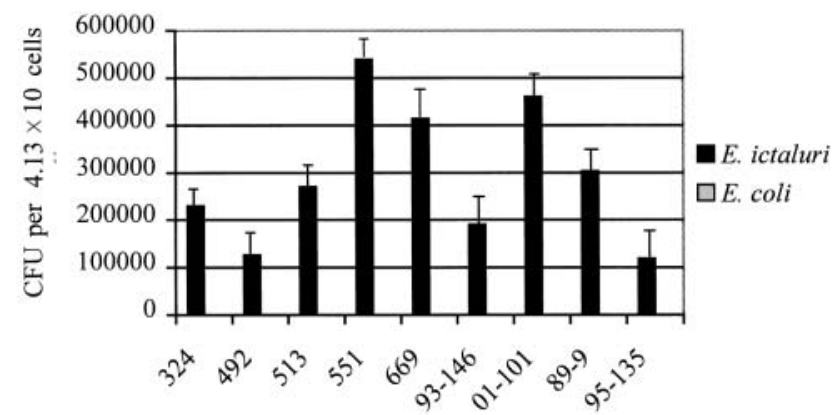

Fig. 3. Colony-forming units (CFU) per monolayer of FHM cell lysate after incubation for $2 \mathrm{~h}$ at $26^{\circ} \mathrm{C}$ with various isolates of Edwardsiella ictaluri (MOI 20 to 50) followed by $4 \mathrm{~h}$ of incubation in medium containing $100 \mu \mathrm{g} \mathrm{ml}^{-1}$ gentamicin. Columns represent means (+SD) of 3 independent experiments performed in triplicate

known. The use of cultured epithelial cells as invasion model systems was attempted due to the difficulties encountered in generating a primary catfish intestinal epithelial cell line. Although differences in surface receptors and biologic functions are expected between fish and mammalian epithelial cells, data gathered using mammalian cells may help identify and define fundamental process(es) involved in E. ictaluri invasion.

In vivo studies show that Edwardsiella ictaluri is rapidly internalized through epithelial-lined membranes and no damage to mucosal epithelial cells is seen. This supports the hypothesis that E. ictaluri uses a surface-exposed ligand recognized by host-cell receptors to facilitate internalization. This is a common theme among intracellular members of the Enterobacteriaceae, as most identified ligands are surfaceexposed or locally secreted proteins. Whether E. ictaluri surface-exposed protein(s) are solely respon- 
sible for internalization or act as ligands for adherence only, and as such are the first of more complicated bacterial-host cell interactions, remains to be determined.

Invasion efficiencies differed between cultured mammalian, fish and harvested catfish intestinal cells; this may indicate fundamental differences in surfaceexposed receptors (quantitative or qualitative), or may be a consequence of the harvesting techniques and MOI differences used. In any case, direct comparison between cell types was not the focus of this work. Moreover, receptors utilized by one cell type may differ from those utilized by other cell types. Details of the specific mechanisms employed by Edwardsiella ictaluri to invade different cell types remain to be defined using these specific in vitro systems.

The low invasion efficiency seen with the enteric cell-suspension assay may be due to an inherent low virulence of the isolate used (LA 89-9) or may be a consequence of the tissue treatment and harvest procedures. To determine if low invasion was due to isolate virulence, 8 other isolates of Edwardsiella ictaluri were placed on FHM cells to determine their invasion efficiencies in comparison to LA 89-9. Although a weak invader of mammalian cell lines and catfish-harvested cells, LA 89-9 was one of the more invasive isolates on the FHM cell line. Hence, the poor invasion seen with harvested cells is likely to be related to the use of trypsin/pepsin to harvest the cells. Trypsin/pepsin may cleave critical host-cell receptors exposed on the surface of epithelial cells, thus lowering the number of available sites for bacterial adherence.

Using chemical blockers, the importance of cellular microfilaments and receptor-mediated endocytosis in uptake of Edwardsiella ictaluri was demonstrated using mammalian epithelial cells. Actin microfilaments and receptor-mediated endocytosis are involved in the uptake of several bacterial species by non-professional phagocytes (Finlay \& Falkow 1989, Finlay et al. 1989). For example, microfilament inhibitors and deactivators of receptor-mediated endocytosis block the entrance of Yersinia and Salmonella species into epithelial cells lines (Finlay \& Falkow 1988, 1989, Finlay et al. 1989, 1991). Based on the data presented here, invasion mechanisms utilized by E. ictaluri are suspected to be similar. Cytochalasin D specifically binds to actin and causes microfilament depolymerization, which results in altered cell morphology and interference with bacterial adherence and entry (Ewanowich \& Peppler 1990). Consequently, intracellular invasion by bacteria requiring surface alterations to facilitate either adherence and/or internalization is substantially reduced.

Microfilaments are also necessary for efficient internalization of clathrin-coated vesicles in receptormediated endocytosis (Gottleib et al. 1993, Lamaze et al. 1997). Since inactivating microfilament function may produce secondary effects on clathrin-coated vesicle internalization, it is unclear if cell surface rearrangements are truly involved in invasion of cells by Edwardsiella ictaluri. As an example, Yersinia enterocolitica grown at $26^{\circ} \mathrm{C}$ expresses the protein invasin, which mediates bacterial uptake via receptormediated endocytosis. Blocking uptake of $Y$. enterocolitica using cytochalasin D most probably represents a secondary effect on receptor-mediated endocytosis. Blocking invasion of E. ictaluri using cytochalasin D may be similar. However, a separate pathway involving altered cell-surface structure mediated by actin rearrangement must also be considered. In addition, the possibility of a complex system of interactions for internalization is also possible.

Optimal MOI is often a critical factor in invasion efficiency. Invasion efficiency of bacteria can be limited by the presence of overwhelming bacterial products, bacterial numbers in the system, or the number of available receptors exposed on epithelial cells (especially important in the case of chemically harvested cells). Although poorly invasive using harvested host cells, Isolate LA 89-9 was one of the best invaders using the FHM cell line, implicating either the MOI or receptor loss as the problem in the suspension cell assay. Trypsin/pepsin may alter surface receptors, interfering with the interaction of bacteria and host cells. Interestingly, Edwardsiella ictaluri isolates with the best invasion capabilities (FHM cell line) were isolated from fish infected during natural outbreaks of ESC. Differences in invasion efficiency among $E$. ictaluri isolates in this study may be a direct consequence of the expression of specific virulence factors that have yet to be identified.

With the increase in resistance of Edwardsiella ictaluri to antibiotics approved for use in food fish, the development of mechanisms to prevent bacterial adherence and infection of host cells is critical. A fuller understanding of these mechanisms and of the interactions that occur between bacterium and host cells will assist future strategies for prophylactic or therapeutic intervention of E. ictaluri infection. These studies identify several susceptible cell lines and a natural host-cell system that function as in vitro models. These cell models will aid in clarifying invasion mechanisms used by E. ictaluri at the cellular and molecular level.

Acknowledgements. Appreciation is extended to Dr. Philip Mixter (Washington State University at Pullman) for kindly providing the Henle 407 cell line, Dr. Joseph Newton for providing channel catfish and bacterial isolates used in this study, and Dr. John Hawke for providing additional Edwardsiella ictaluri isolates tested. This work was supported by the United States Department of Agriculture, NRI No. 00-352049211. 


\section{LITERATURE CITED}

Baldwin TJ, Newton JC (1993) Pathogenesis of enteric septicemia of channel catfish caused by Edwardsiella ictaluri: light and electron microscopic and bacteriologic findings. J Aquat Anim Health 5:189-198

Blazer VS, Shotts EB, Waltman WD (1985) Pathology associated with Edwardsiella ictaluri in catfish, Ictalurus punctatus Rafinesque and Danio devario. Fish Biol 27:167-175

Cooper RII (1996) Use of a mini-transposon to study chondroitinase activity associated with Edwardsiella ictaluri. J Aquat Anim Health 8:319-324

Ellinghorst EA (1994) Measurement of invasion by gentamicin resistance. Methods Enzymol 236:405-420

Ewanowich CA, Peppler MS (1990) Phorbol myristate acetate inhibits HeLa 229 invasion by Bordatella pertussis and other invasive bacterial pathogens. Infect Immun 58: 3187-3193

Finlay BB, Falkow S (1988) A comparison of microbial invasion strategies of Salmonella, Shigella and Yersinia species. In: Horowitz MA (ed) Bacterial-host cell interactions, Alan R Liss, New York, p 227-243

Finlay BB, Falkow S (1989) Common themes in microbial pathogenicity. Microbiol Rev 53:210-230

Finlay BB, Falkow S, Heffron F (1989) Epithelial cell surfaces induce Salmonella proteins required for bacterial adherence and invasion. Science 243:940-943

Finlay BB, Ruschkowski S, Dedhar S (1991) Cytoskeletal rearrangements accompanying Salmonella entry into epithelial cells. J Cell Sci 99:283-296

Gottleib TA, Ivanov IE, Adesnik M, Sabatini DD (1993) Actin microfilaments play a critical role in endocytosis at the apical but not the basolateral surface of polarized epithelial cells. J Cell Biol 120:695-710

Hawke JP (1979) A bacterium associated with disease of pond cultured channel catfish (Ictalurus punctatus). J Fish Res Board Can 36:1508-1512

Hawke JP, McWhorter AC, Steigerwalt AG (1981) Edward-

Editorial responsibility: David Bruno,

Aberdeen, Scotland, UK siella ictaluri sp. nov., the causative agent of enteric septicemia of catfish. Int J Syst Bacteriol 31:396-400

Lamaze C, Fujimoto LM, Yin HL, Schmid SL (1997) The actin cytoskeleton is required for receptor mediated endocytosis in mammalian cells. J Biol Chem 272:203-325

Miller VL, Finlay BB, Falkow S (1988) Factors essential for penetration of mammalian cells by Yersinia. Curr Top Microbiol Immunol 138:15-39

Mitchell AJ (1997) Fish disease summaries for the southeastern United States from 1976-1995. Aquac Mag 23:87-93

Miyazaki T, Plumb JA (1985) Histopathology of Edwardsiella ictaluri in channel catfish, Ictalurus punctatus (Rafinesque). J Fish Dis 8:389-392

Newton J, Bird C, Blevins WT (1988) Isolation, characterization and molecular cloning of cryptic plasmids isolated from Edwardsiella ictaluri. Am J Vet Res 49:1856-1860

Oeschlager TA, Barrett TJ, Kopeck JT (1994) Some structures and processes of human epithelial cells involved in uptake of enterohemorrhagic E. coli 0157:H7 strains. Infect Immun 62:5142-5150

Pastan IH, Willingham MC (1981) Receptor mediated endocytosis of hormones in cultured cells. Annu Rev Physiol 43: 239-250

Plumb JA, Vinitnantharat S (1990) Dose titration of Sarafloxicin (A-56620) against Edwardsiella ictaluri infection in channel catfish. J Aquat Anim Health 2:194-197

Rubens CE, Smith S, Hulse M, Chi EY, van Belle G (1992) Respiratory epithelial cell invasion by group B streptococci. Infect Immun 60:5157-5163

Saeed MO, Plumb JA (1986) Immune response of channel catfish to lipopolysaccharide and whole cell Edwardsiella ictaluri vaccines. Dis Aquat Org 2:21-25

Shotts EB, Waltman WD (1990) A medium for the selective isolation of Edwardsiella ictaluri. J Wildl Dis 26:214-128

Shotts EB, Blazer VS, Waltman WD (1985) Pathogenesis of experimental Edwardsiella ictaluri infections in channel catfish (Ictalurus punctatus). Can J Fish Aquat Sci 34: $35-42$

Submitted: August 20, 2001; Accepted: November 13, 2001 Proofs received from author(s): August 27, 2002 